\begin{tabular}{l|l} 
REVISTA & $\begin{array}{l}\text { Revista Educación } \\
\text { ISSN: 0379-7082 } \\
\text { ISSN: 2215-2644 } \\
\text { revedu@ @ gmail.com } \\
\text { Universidad de Costa Rica } \\
\text { Costa Rica }\end{array}$
\end{tabular}

\title{
Resiliencia y coevolución neuroambiental
}

\author{
Carazo Vargas, Viviana \\ Resiliencia y coevolución neuroambiental \\ Revista Educación, vol. 42, núm. 2, 2018 \\ Universidad de Costa Rica, Costa Rica \\ Disponible en: http://www.redalyc.org/articulo.oa?id=44055139033 \\ DOI: https://doi.org/10.15517/revedu.v42i2.28137
}

Esta obra está bajo una Licencia Creative Commons Atribución-NoComercial-SinDerivar 3.0 Internacional. 


\section{Resiliencia y coevolución neuroambiental}

Resilience and Neuro-Environmental Coevolution

Viviana Carazo Vargas [1]

Universidad de Costa Rica, Costa Rica

viviana.carazo@ucr.ac.cr
DOI: https://doi.org/10.15517/revedu.v42i2.28137

Redalyc: http://www.redalyc.org/articulo.oa?id=44055139033

Recepción: 08 Marzo 2017

Aprobación: 22 Junio 2018

\section{Resumen:}

La resiliencia como constructo social plantea un panorama conceptual confuso y diverso el cual incluye su visualización como cualidad individual, como rasgo colectivo, o como proceso dinámico, entre otros, con el común denominador de la adaptación que se genera ante la vivencia de una situación retadora. En la exploración histórica de los orígenes de este concepto, se evidencia cómo las ciencias sociales han tendido a atribuir la mayor parte de la responsabilidad del proceso adaptativo, al individuo en sí mismo más que a las relaciones que este establece con la multiplicidad de eventos y factores que experimenta a lo largo de su vida. El presente ensayo analiza esta situación y propone la consideración de la resiliencia como un proceso coevolutivo y dinámico de aprendizaje, no solo del individuo sino también del entorno con el cual se encuentra en estrecha coevolución. Se concluye resaltando la importancia de considerar la evidencia neurocientífica actual para analizar la resiliencia como parte de un proceso neurobiológico y coevolutivo que permite la diversidad, la supervivencia de la especie y la exploración y comprensión de la afectación mutua entre los organismo y sus entornos.

Palabras Clave: resiliencia, desarrollo humano, aprendizaje, coevolución.

\section{Abstract:}

Resilience as a social construct raises a confused and diverse conceptual panorama that includes its visualization as an individual quality, as a collective feature, or as a dynamic process, among others, with the common denominator of the adaptation that is generated in the face of a challenging situation. In the historical exploration of the origins of this concept, it is evident how the social sciences have tended to attribute the greater part of the responsibility of the adaptive process, to the individual in itself more than to the relations that this establishes with the multiplicity of events and factors that are experienced throughout life. The present essay analyzes this situation and proposes the consideration of resilience as a coevolutionary and dynamic process of learning, not only of the individual but also of the environment with which it is in close coevolution. The essay is concluded by highlighting the importance of considering current neuroscientific evidence to analyze resilience as part of a neurobiological and coevolutionary process that allows diversity, the survival of the species and the exploration, and the understanding of the mutual affectation between organisms and their environments.

KEYWORDS: resilience, human development, learning, coevolution.

\section{INTRODUCCIÓN}

En este punto de la historia, la reiteración no es un agravante: no existe base científica para el determinismo. Desde los primeros momentos de vida, organismo y ambiente coevolucionan para hacer de un ser humano,

\section{NotAS DE AUTOR}

[1] Costarricense. Doctora en Neuropsicología Clínica (Universidad de Salamanca, España), Máster en Estudios Interdisciplinarios sobre Discapacidad con Énfasis en Discapacidad Múltiple y Sordoceguera (Universidad de Costa Rica). Licenciada en Retos Múltiples y Bachiller en Ciencias de la Educación con Énfasis en Educación Especial (Universidad de Costa Rica). Ha realizado diversos cursos internacionales de especialización en Neuropsicología Infantil y Trastornos Generalizados del Desarrollo. Laboró para el Ministerio de Educación Pública de Costa Rica, en la Escuela Neuropsiquiátrica Infantil. Ha sido docente en la UNED, en la Universidad Santa Paula y en la Universidad de La Salle. Su trabajo investigador y publicaciones han sido en el ámbito del neurodesarrollo y la neuropedagogía. Actualmente se desempeña como profesora e investigadora en la Escuela de Orientación y Educación Especial de la Universidad de Costa Rica, ejerce como Punto Focal en Educación en Representación de la Academia Nacional de Ciencias de Costa Rica ante la Interamerican Network Academies of Sciencies (IANAS), además se dedica a la evaluación, asesoramiento e intervención Neuropsicopedagógica y Conductual en niños y adolescentes. 
lo que es en cada instante de su historia (Carazo y López, 2009). Cada hebra de ADN se expresará de modo diferente en función de la estructura celular que rodea al código genético (Jablonka y Lamb, 2002); de igual forma, entorno interno y externo incidirán en los procesos de proliferación, migración y diferenciación neuronal, en el establecimiento de conexiones sinápticas, en la apoptosis selectiva y en la estabilización de circuitos de un organismo esencialmente plástico (Gottesman y Hanson, 2005). Sanes, Reh, y Harris, 2000). Son también los rasgos genéticos y epigenéticos, los que además de hacer única a cada persona, le proporcionarán la posibilidad de desarrollar sistemas para percibir el entorno, formar memorias, aprender, modelar sus estados mentales, neuropsicológicos y cognitivos superiores, y así, zambullirse en un mundo de símbolos, signos y significados, influir en los otros, y ser influido por ellos.

La coevolución, planteada por Carazo y López (2009, p.5) como el "mecanismo y proceso de la evolución mediante el cual el ambiente y el organismo se influyen y modelan mutua y dinámicamente”, constituye un mecanismo natural para la gestación de diversidad, influye en que cada persona sea particular y distinta al resto y al mismo tiempo, representa evidencia de que sea cual sea el color de la piel, la talla, la estatura, el sexo, la modalidad perceptual predominante, el estilo cognitivo o las características conductuales, los seres humanos comparten la capacidad de percibir su entorno, formar sinapsis y aprender (Carazo, 2013).

Ello no quiere decir, de ninguna forma, que se niegue la existencia de condiciones genéticas, estructurales, lesionales o neurodegenerativas que diversifiquen aún más la atención que necesitan algunos seres humanos para aprovechar al máximo su potencial, ni que se desmerezcan las políticas y acciones de prevención y equiparación de oportunidades (nada más alejado de la perspectiva del presente ensayo).

Los alcances de la coevolución entre el organismo y su entorno se conocen cada vez más; el nutrimento que constituye la interacción social y la depauperación sistémica que conlleva la exclusión de las relaciones con los otros, surgen con mayor fuerza como elementos promotores o limitadores de la máxima expresión dinámica del potencial de desarrollo de un ser humano. En un momento de cambios trascendentales en el sistema educativo costarricense (Decreto Ejecutivo 40955), la consideración de este hecho debe estar clara para enriquecer el sustento de las prácticas inclusivas.

Es en este proceso de comprensión, que la resiliencia parece haber reaparecido, inicialmente más como un constructo social revestido de benevolencia que como proceso neuroevolutivo (López, 2005). Sin embargo, si se acepta el hecho de que cualquier conducta y estado mental requiere de actividad cerebral, y que el neurodesarrollo tiene características epigénicas fundamentales, la resiliencia, con las bases neuroevolutivas que por lo tanto ha de tener, representa un avance enriquecedor en el intento de entender los alcances de la mutua afectación que se da entre el individuo y el entorno. Esta es la razón de ser del presente ensayo.

\section{Panorama conceptual de la resiliencia, un paisaje confuso}

$\mathrm{Al}$ intentar abordar el panorama conceptual de la resiliencia, surge una situación particular, no novedosa pero sí importante en el proceso de entendimiento de lo que ha sido un constructo básicamente social: las definiciones acerca de lo que se entiende por resiliencia, son múltiples, muy cercanas a lo que Reghezza-Zitt, Lhomme y Provitolo (2015) describen como un arcoíris de significados.

Por ejemplo, al asumirla desde una perspectiva individual (Van Kessel, 2013) se le considera como una "predisposición del individuo a resistir las consecuencias potencialmente negativas del riesgo y desarrollarse adecuadamente" (Engle, Castle y Menon, 1996, p.622), como "la capacidad de una persona o de un grupo para desarrollarse bien, para seguir proyectándose en el futuro a pesar de acontecimientos desestabilizadores, de condiciones de vida difíciles y de traumas a veces graves" (Manciaux, Vanistendael, Lecomte y Cyrulnik, 2003, p.22), como un "conjunto de cualidades, que fomenta un proceso de adaptación exitosa y transformación en la vida, a pesar de los riesgos y la adversidad" (Benard, 1996, p.2), una "cualidad que caracteriza a niños quienes, habiendo sido expuestos a un estrés y adversidad significativo en sus vidas, no sucumben a los fallos escolares y de vida que les han sido predichos" (Finley, 1994, p.2), como "una cualidad dinámica que se encuentra 
latente en el interior de cada ser humano, cualidad siempre cambiante que surge de la creencia en la propia eficacia para enfrentar los cambios y resolver problemas" (Puerta, 2002, p.14) como "un complejo repertorio de tendencias conductuales" (Agaibi y Wilson, 2005, p.197) o un conjunto de cualidades personales positivas que mejoran la adaptación individual ante circunstancias adversas (Rodríguez-Fernández et ál. 2016).

Otros abordajes varían al conceptualizarla como una capacidad no solo individual sino también colectiva "para desarrollarse, para continuar proyectándose en el porvenir pese a la presencia de acontecimientos desestabilizadores, pese a la presencia de condiciones de vida difíciles o de hechos traumáticos que en ocasiones son graves" (Titran, 2004, p.241), visualizándola también como "la capacidad que tienen niños, adultos o familias, para actuar correctamente y tener éxito pese a las circunstancias adversas que los rodean, para recuperarse después de vivir eventos estresantes y reasumir con éxito su actividad habitual" (Puerta, 2002, p.14.), o como la

"capacidad universal que permite a una persona, un grupo o una comunidad, impedir, minimizar o superar los efectos dañinos de la adversidad... que... no solamente posibilita un desarrollo normal dentro de las condiciones de adversidad, sino que también puede promover un crecimiento más allá del nivel actual de funcionamiento" (Grotberg, 1997, citado por Puerta, 2002, p.14-15).

Sin embargo, en contraste con la conceptualización individual o colectiva, hay autores como Stein, Fonagy, Ferguson y Wisman (2000, p.287) quienes anotan que

\footnotetext{
"dentro de una perspectiva de desarrollo, la resiliencia no es algo que el niño simplemente posea. Es la capacidad de rehacer o rebotar, sobreponerse tras un período difícil, (o durante situaciones de mucho estrés) implicada a lo largo del tiempo dentro del contexto total de las influencias ambientales. La capacidad para mantenerse organizado en períodos de reto o cambio, afrontar las situaciones activamente y mantener expectativas positivas durante períodos de estrés que involucran a la persona en interacción con el ambiente a través de sucesivos períodos de adaptación... una capacidad no estática, sino continuamente influenciada por los cambios emergentes en el ambiente".
}

Esta visión de dinamismo, considera que cada individuo cambia a lo largo del tiempo en respuesta a las experiencias de vida. Bell, Romano y Flynn (2015) también definen a la resiliencia como la capacidad de un sistema dinámico, de recuperarse de retos significativos a su estabilidad o desarrollo.

En los últimos años, investigaciones como la de Lavoie, Pereira y Talwar (2016) destacan el papel del entorno y su influencia sustancial en los factores psicológicos y físicos que inciden en la resiliencia del individuo.

Otros investigadores han definido a la resiliencia destacando el elemento de adaptación, visualizándola como un "proceso que incluye una adaptación positiva dentro de un contexto de adversidad significativa" (Luthar, Cicchetti y Becker, 2000, p.543), como un afrontamiento efectivo y adaptativo a pesar de enfrentarse con la pérdida, la dificultad o la adversidad, es decir, como la "habilidad para rebotar de las experiencias emocionalmente negativas y adaptarse flexiblemente a las demandas cambiantes de las experiencias estresoras" (Turgade y Fredrickson, 2004, p.320), como el "proceso de vencer los efectos negativos de la exposición al riesgo, afrontando exitosamente las experiencias traumáticas y evitando las trayectorias negativas asociadas con los riesgos" (Fergus y Zimmerman, 2005, p.399) y como un indicador de una adaptación exitosa en el contexto del proceso de desarrollo de una persona (Dubowitz, et ál. 2016).

Se le ve también como un "proceso común que resulta en la mayoría de los casos de la acción de sistemas adaptativos básicos, lo que no descarta que en determinadas situaciones, un talento particular o un apoyo externo, juegue un papel importante ", o, "un proceso ordinario caracterizado por un buen resultado a pesar de serios riesgos para la adaptación o el desarrollo" (Masten, 2001, p.228), sin embargo también existen perspectivas contrarias como la de Robinson (2000), quien considera que el contemplar la resiliencia como un proceso "ordinario" no es el enfoque adecuado, pues afecta el establecimiento de estrategias preventivas.

Y también es posible mencionar, a manera de ejemplo, perspectivas como la de Born y Böet (2004), quienes anotan que la resiliencia es básicamente un concepto, o como la de Manciaux (2004), que considera que la 
resiliencia es un concepto original y no simplemente un atavío nuevo para nociones antiguas; que no es una resistencia corregida y aumentada, ni invulnerabilidad o un afrontamiento mejorado y que sostiene que la resiliencia está compuesta por dos facetas inseparables: por un lado, la destrucción, el descalabro; por otro la reconstrucción, la dinámica existencial, el proyecto de una vida nueva o de una vida mejorada y reanudada.

El horizonte parece complicarse aún más, cuando se identifican abordajes que se refieren a la existencia de "tipos" de resiliencias. Por ejemplo, en la hipótesis de Born y Boët (2004), consideran que no existe una resiliencia común a todos los individuos ni a todas las situaciones, sino más bien varias resiliencias, cada una de ellas específicas respecto a un conjunto de factores de riesgo.

Por su parte, Lawford y Eiser (2001) dividen a la resiliencia en dos subtipos: el asociado con la resistencia al estrés, y el que se refiere a la recuperación de un trauma. Rak (2002) hace referencia a tres tipos de resiliencia: la que se asocia a un desarrollo positivo a pesar de experiencias de alto riesgo, la que se refiere a la presencia de un desarrollo competente a pesar de situaciones agudas de riesgo, y la que hace referencia a la recuperación ante un trauma. Kim-Cohen, Moffitt, Caspi y Taylor (2004) hablan de resiliencia conductual y de resiliencia cognitiva. Tugade y Fredrickson (2004) se refieren a la resiliencia psicológica. Bosworth y Earthman (2002) comentan la existencia de resiliencia a corto y a largo plazo. Fine (1991) identifica dos fases dentro de lo que en su trabajo se define como resiliencia (una aguda y una de reintegración), y en su investigación, Fergus y Zimmerman (2005) mencionan tres modelos de resiliencia: el compensatorio, el de factores protectores (que puede a su vez, ser estabilizador o reactivo) y el modelo de retos; por su parte Walker et ál. (2017) aborda una perspectiva ampliamente estudiada en los últimos años, la "resiliencia psicológica".

Esto, sin mencionar los numerosos instrumentos, escalas y listas de cotejo que han sido desarrolladas para medir y/o evaluar la resiliencia, y que contrastan con posiciones como la de Lawford y Eiser (2001) que sostienen que un constructo latente no puede medirse directamente, pues no es posible cuantificar un fenómeno que no es directamente observable sino que solo puede ser inferido.

Las investigaciones acerca de la resiliencia, son igualmente numerosas y diversas, basta con echar un vistazo en los buscadores informáticos o en las principales bases de datos.

Entonces... ¿de qué se habla cuando se hace referencia a la resiliencia?, ¿es esta una cualidad, un concepto o un proceso?, ¿constituye una variable dependiente o independiente?, ¿existe realmente la resiliencia? Luthar, Cicchetti y Becker (2000) analizan el panorama y sin duda se concuerda con ellos en que la investigación muestra poco consenso sobre la definición a utilizar, así como variaciones importantes en la operacionalización y estudio de los constructos que para cada quien implica (aunque parece coincidirse al señalar una situación de riesgo o de adversidad y un cambio en la posterior adaptación).

Otra situación que surge, es la indiscutible carga subjetiva que suelen llevar las definiciones, palabras como "bien", "difícil", "grave", "exitoso", "correcto", "adverso", "normal", "activo", "positivo", "negativo", "bueno" suelen ser adjetivos frecuentes cuya interpretación es sin duda dependiente de la experiencia de vida de cada investigador.Tomkiewicz (2004) considera que la inclusión de condiciones morales y sociales, así como en qué contexto y cómo, se da la resiliencia, están muy ligados al uso del concepto, lo que se ilustra en que los requisitos para la estimación de la resiliencia han variado desde la consideración de la oblación, a la "moralidad", el ser "socialmente correcto", hasta la observación de Hunter y Chandler (1999), quienes al estudiar a un grupo de adolescentes identificaron que en su caso, el ser resiliente no era ni tener un "sano" concepto de uno mismo, ni un fuerte sentido de autovaloración, ni la habilidad de "conectar" y confiar en los otros; sino que ser resiliente, para los sujetos de su estudio, significaba sobrevivir.

El panorama actual en cuanto a la identificación de lo que constituye la resiliencia, continúa siendo mejor entendido bajo la observación de Luthar, Cicchetti y Becker (2000, p.555) (de la cual se hace eco), en relación con que "la investigación en resiliencia, carece notablemente de prestar atención al papel de la biología, no solo al cómo los procesos biológicos afectan los procesos psicológicos, sino además, cómo las experiencias psicológicas pueden modificar la estructura y funcionamiento cerebral". Ante dicha dicotomía, el juicio personal de lo que es considerado o no, resiliencia, puede ser fácilmente cargado de prejuicios morales y variar 
ampliamente según la perspectiva de quien haga referencia al tema. En opinión de Luthar, Cicchetti y Becker (2000), el progreso en el estudio y dilucidación conceptual de la resiliencia, va a continuar viéndose limitado en tanto las investigaciones sigan siendo manejadas únicamente por la experiencia empírica y no se parta de una teoría seria acerca del desarrollo humano.

\section{EXPLORACIÓN DE LOS ORÍGENES DEL CONSTRUCTO, UN INTENTO POR COMPRENDER SU UTILIZACIÓN HISTÓRICA}

En procura de comprender de qué es lo que se habla cuando se hace referencia a la resiliencia, y conseguir llegar más adelante a la perspectiva que se asume en el presente ensayo, se considera importante realizar una breve exploración de los orígenes de este concepto. Se dice que, desde el punto de vista etimológico, resiliencia proviene de los términos latinos "salire" y "resilio", que se traducen como saltar hacia atrás, rebotar o ser repelido, y que el prefijo re, indica reanudación. Por otra parte, sus raíces en el francés antiguo se refieren a "resiliar un contrato", o reanudar el desarrollo de algo pese a la ocurrencia de un incidente que lo ha afectado (Pedrot, 2004; Poilpot, 2004; Reghezza-Zitt, Lhomme y Provitolo, 2015; Theis, 2003).

\subsection{Metalurgia, siderurgia y osteología}

El concepto de resiliencia empezó a ser aplicado al desarrollo humano desde hace pocos años. Sin embargo, no es nuevo en la historia. Vanistendael (1994) citado por Puerta (2002) menciona que lo que implica el concepto de resiliencia es tan antiguo como la misma humanidad. Es un constructo utilizado por largo tiempo en la metalurgia, en la ingeniería y en la mecánica en donde también ha tenido usos distintos. Desde estas disciplinas, se ha empleado para referirse a la capacidad de ciertos materiales o metales de resistir un impacto y recuperar su estructura, su equilibrio interno. De igual forma, se ha considerado que, en este ámbito, la resiliencia implica la recuperación de la estructura interna molecular después de que un material ha sido sometido a grandes presiones y altas temperaturas.

Por otra parte, en la siderurgia, tecnología relacionada con la producción del hierro y sus aleaciones, en especial las que contienen un pequeño porcentaje de carbono, y que constituyen los aceros, se encuentra otro aporte histórico a las raíces del concepto de resiliencia.

El hierro es un material que se corroe rápidamente, en su estado original es altamente quebradizo y se rompe con facilidad; y el carbón por su parte, un mineral orgánico negro y combustible, es también poroso, frágil y requiere de procesos de descomposición, destilación, aleación u otros para la obtención de materiales funcionales para el uso humano. Ambos elementos, en estado puro y por separado, no reportan mayores utilidades, sin embargo la aleación del hierro y el carbono, mediante la intervención de un agente externo que los une en cantidades determinadas, a temperaturas específicas y durante períodos también definidos, da como resultado el acero, un material con características resistentes, de gran dureza pero a la vez plástico y dúctil, es decir, que puede soportar fuerzas considerables, sufrir grandes deformaciones, estiramientos y presiones sin quebrarse (Olsson, Bond, Burns, Vella-Brodrick y Sawyer, 2003; Reghezza-Zitt, Lhomme y Provitolo, 2015; Turgade y Fredrickson, 2004). En este sentido, la resiliencia implica más bien una transformación y no el retorno a la estructura original.

El término de resiliencia también es utilizado en medicina, en donde la osteología lo adopta para expresar la capacidad de los huesos, luego de sufrir una fractura, de unirse, soldarse y adquirir mayor fortaleza en el punto de unión. En dicho caso, usualmente un agente externo interviene para inmovilizar la zona y facilitar la unión de los extremos del hueso, creando un punto de fusión que resulta incluso más fuerte que las otras zonas del hueso. De esta manera, la resiliencia tiene en sí, el mismo trasfondo: la transformación (Pedrot, 2004). 
Impresiona que al realizar la aplicación del concepto a las ciencias sociales, inicialmente se atribuyó más importancia a la sustancia básica, a la cualidad de un material en sí mismo luego de determinado proceso, más que a la sucesión de eventos mediante los cuales este adquiría las propiedades posteriores, y es quizá esta perspectiva, la que ha predominado en muchos aspectos hasta nuestros días.

\subsection{Aplicación en las ciencias sociales}

Se dice que en las ciencias sociales, el término resiliencia empezó a ser utilizado en relación con los seres humanos, a raíz del trabajo que realizaron las psicólogas Emmy Werner y Ruth Smith. Estas estadounidenses iniciaron en 1955 un estudio longitudinal en Hawái, específicamente en la isla Kauai dando seguimiento durante 40 años a una cohorte de 698 personas (todos los niños nacidos en la isla en 1955). En su investigación, monitorearon el impacto de factores de riesgos biológicos, psicológicos y sociales, desde el periodo perinatal, y a los 1, 2, 10, 18, 31-32 y 40 años. Desde 1967 empezaron a publicar sus resultados, identificando a través de los años, a un porcentaje de personas que habiendo atravesado por serios y múltiples problemas, lograron una "vida plena de sentido" y llegaron a ser adultos "seguros de sí, competentes y caritativos" (Werner et ál. 1968; Werner, Honzik y Smith, 1968; Werner, 2004). Cuando los investigadores se enfocaron en estudiar las competencias de estos individuos, sus capacidades para el afrontamiento y sus estrategias de ajuste, nació el modelo o constructo de la resiliencia aplicado al desarrollo humano. En la actualidad, se considera que su trabajo fue un elemento que favoreció la adopción en el mundo anglosajón, del concepto de resiliencia dentro de las ciencias humanas (Luthar, Cicchetti y Becker, 2000; Puerta, 2002; Theis, 2003; Tomkiewicz, 2004).

A partir de ahí, la historia de la investigación en resiliencia empezó a desarrollarse bajo dos enfoques principales (Jacelon, 1997; Reghezza-Zitt, Lhomme y Provitolo, 2015; Van Kessel, 2013): como rasgo del individuo o característica de personalidad que modera los efectos negativos de la exposición a la adversidad y promueve la adaptación, o como un proceso dinámico producto de complejas interacciones entre la persona y su medio.

\subsubsection{Invulnerabilidad y resiliencia como rasgo individual}

En la década de 1970, al tomar mayor fuerza el área de investigación de niños considerados de alto riesgo, y en un intento por comprender cómo y en qué grado los riesgos afrontados modelan la vida de un ser humano, el francés Koupernik y el estadounidense Anthony introdujeron el concepto de invulnerabilidad, que habría de tener gran influencia en la concepción de la resiliencia en años posteriores (Lavoie, Pereira y Talwar, 2016; Proag, 2014; Theis, 2003).

El estudio de las vulnerabilidades e inmunidades, de las defensas, competencias y capacidades asociadas al enfrentamiento de una situación de adversidad, fue el que marcó la pauta en este sentido. De hecho, las primeras ideas acerca de la resiliencia incluían descriptores como "invulnerables", "invencibles" o "superniños" en relación con aquellos quienes salían adelante de experiencias catalogadas como difíciles para quienes les estudiaban.

Las investigaciones bajo el enfoque de la invulnerabilidad, se dirigían específicamente a las características personales; es decir, la resiliencia era conceptualizada como el resultado de rasgos de personalidad o estilos de afrontamiento que parecían hacer que algunas personas continuaran progresando a lo largo de una "trayectoria positiva de desarrollo", aun cuando fueran confrontados con una adversidad considerable (Finley, 1994). Este enfoque, contenía implícita la perspectiva de que el esperar cualidades extraordinarias en los individuos considerados resilientes, implicaba que los recursos y sistemas ordinarios adaptativos no eran 
suficientes, sino que había personas que tenían un "algo más", que les hacía sobresalir de situaciones difíciles (Lavoie, Pereira y Talwar, 2016; Masten, 2001; Olsson, Bond, Burns, Vella-Brodrick y Sawyer, 2003).

Con la perspectiva de la existencia de la invulnerabilidad, de unos seres humanos invencibles, surgió una de las líneas de investigación en resiliencia que ha calado más hondo en el área. Como pioneros al respecto, Block y Block han venido trabajando desde los años cincuenta en lo que han llamado la "ego resiliencia". Es posiblemente una de las primeras utilizaciones del concepto de la resiliencia en la psicología propiamente. La definen como "la capacidad dinámica de un individuo para modificar su nivel modal de ego control, en cualquier dirección, en función de las demandas características del contexto ambiental". (Block y Block, 1980, p.48). Su enfoque de la resiliencia como una adaptación ingeniosa del individuo a las circunstancias ambientales, mediante un uso flexible de las estrategias de resolución de problemas, la coloca en una situación de relación directa con la regulación de la personalidad o el ego, e incluye además la percepción de la "baja resiliencia" como poca flexibilidad adaptativa de la persona, inhabilidad para responder a las circunstancias cambiantes, tendencia a perseverar y dificultad para recuperarse después de una experiencia difícil (Eisenberg et ál. 2004).

En 1996, Block y Kremen reafirman su posición y destacan que la "ego resiliencia" mantiene un grado importante de asociación con la inteligencia (a la cual consideran, todavía en estas fechas, como el puntaje obtenido en una prueba de CI), sosteniendo que lo que en estos momentos se denomina resiliencia, es solo una "jerga contemporánea" de lo que en la antigua psicología se denominaba "ego fuerte" y que relacionan también con lo que ha sido llamada "inteligencia social".

Esta perspectiva, de la resiliencia como rasgo estrictamente personal, ha encontrado eco en múltiples líneas de investigación, y es posible apreciarla en trabajos que la definen como "la capacidad que tienen algunas personas... que poseen algunos supervivientes... o algunas víctimas" para "encajar el golpe... soportar su suerte... sobrevivir" (Vaillant, 2004), en aquellos que hablan de personas resilientes y personas no resilientes, dependiendo de su capacidad para resistir, responder o desarrollar efectividad ante situaciones adversas, en los que centran las posibilidades de adaptación y competencia resiliente que se desarrollen, únicamente en los rasgos personales (Mandleco y Peery, 2000), o que la resaltan como capacidad individual, natural e innata (Kelley, 2005).

Los cuestionamientos no se hicieron esperar, entre ellos la observación de Luthar, Cicchetti y Becker (2000), quienes destacan que existe una confusión terminológica y que la ego resiliencia es una característica personal del individuo, pero que la resiliencia en sí misma, constituye un proceso dinámico de desarrollo. Estos investigadores comentan que cualquier representación científica de la resiliencia como rasgo personal, puede inadvertidamente llevar a la percepción de que algunos individuos, simplemente no cuentan con ese rasgo.

Otras críticas son comentadas por Waller (2001) al señalar que el énfasis en la invulnerabilidad individual es problemático, primero porque básicamente, es contrario a la tesis de la condición humana, segundo, porque hace sumamente difícil comprender que el patrón de desarrollo de alguien pudiera identificarse como resiliente en algunos sentidos, y vulnerable en otros, y tercero, porque al centrarse en los factores personales, se oscurecía el análisis del contexto ecosistémico de la resiliencia, un enfoque que también empezaba a tomar fuerza.

Por otra parte, Waller (2001) agrega que con la idea de la existencia de una invulnerabilidad particular, se corre el riesgo de caer en una visión despreciativa de algunos individuos, familias y comunidades, viéndoles como deficientes, débiles y culpables cuando no logran solucionar sus problemas por sí mismos, marcando así la tendencia a patologizar el sufrimiento como "culpa de la víctima", y señalándolo para representar los problemas sociales de algunos sectores como su propia culpa más que reconocer la responsabilidad de la sociedad en la formación y mantenimiento de estos.

Observaciones como la de Tomkiewicz (2004), también señalan que la invulnerabilidad, al igual que el enfrentamiento en sí mismo, solo significan resistencia, y por lo tanto una respuesta inmediata y de cierta 
manera estática, pero que la resiliencia, por el contrario, implica un efecto duradero, un dinamismo y un proyecto de vida.

En muchos sentidos, la perspectiva de invulnerabilidad concordaba con la visión determinista que dominaba en diversos sectores; concepto que empezaría a cuestionarse y dejarse de lado en muchos ámbitos investigativos conforme surgió el reconocimiento de la interacción entre organismo y entorno como elemento confluyente e influyente en la vida de un ser humano.

Sin embargo, como se mencionó, esta ha sido solo una de las perspectivas de la resiliencia; Rutter (1987) citado por Waller (2001) comenta que el estudio de este constructo está evolucionando de conceptualizaciones estáticas e individualistas hacia una apreciación de la complejidad de los aspectos relacionales y contextuales implicados en la adaptación ante la adversidad. Uno de los puntos que han sido rescatados de la perspectiva de la resiliencia como rasgo individual, es el potencial que tiene cualquier ser humano, de interactuar con diversos factores o situaciones que reduzcan o alteren las consecuencias de la exposición a condiciones adversas, y promuevan la expresión dinámica del máximo potencial de desarrollo. Waller (2001) destaca que un paso esencial en este proceso es adoptar un enfoque respetuoso y colaborador, para el reconocimiento y construcción de los recursos existentes en el individuo, la familia, la comunidad y la cultura.

\subsubsection{Resiliencia como proceso dinámico y coevolutivo}

El cuestionamiento del concepto de invulnerabilidad, incidió también en un reconocimiento de cómo la relación entre el organismo y su entorno confluye para modelar la vida de un ser humano.

Esta perspectiva de la resiliencia como un proceso dinámico producto de complejas relaciones entre la persona, su familia, comunidad y el entorno social y cultural, fue impulsada por Michael Rutter, uno de los mayores investigadores en el tema de la resiliencia. En su modelo plantea que la respuesta individual ante una situación de adversidad va a ser determinada por la interacción dinámica entre una constelación de variables consideradas a través del tiempo, en donde las experiencias son parte de ese dinamismo que incide en las relaciones que se desarrollan con el entorno. Este investigador afirma que la resiliencia no es absoluta, que tiene límites y que su origen nunca es puramente intrínseco y además, que "es variable en función de los riesgos, variable en el tiempo y siempre de origen simultáneamente intrínseco y epigenético" (Rutter, citado por Tomkiewicz, 2004, p.38).

Bajo este enfoque, se ubica a muchos de los trabajos anglosajones de los últimos años, en los que al igual que Manciaux, Vanistendael, Lecomte y Cyrulnik (2003) ya consideran a la resiliencia, como una capacidad potencial que se desarrolla bajo el reconocimiento y estimulación de la interacción constante con el ambiente, dejando de lado al determinismo, el cual no tiene cabida ni sentido al ampliar los horizontes de las ideas con respecto a los procesos de desarrollo humano. Bouvier (2003, p.72) lo ilustra con claridad: "la resiliencia nos muestra a la vez, que lo esencial no es lo constitucional ni lo ambiental, sino una interacción activa entre ambos... el papel activo que tienen el niño y su entorno, contradice el determinismo".

Trabajos como el de Kim-Cohen, Moffitt, Caspi y Taylor (2004) ilustran la progresiva profundización en los factores que intervienen en las respuestas ante una situación adversa, ellos destacan la posibilidad de la participación de elementos genéticos, además de aspectos familiares y del ecosistema más amplio; de igual forma, Tarter y Vanykov (1999) sugieren que

"el estudio de la genética de la conducta y el reconocimiento de las diferencias individuales como algo que resulta de la interacción y de la adecuación entre fenotipo y ambiente, ofrecen explicaciones más profundas sobre la forma que puede utilizar el individuo para precaverse de las agresiones estresantes" (Tarter y Vanykov, 1999, citados por Mayhew y Mayhew, 2003, p.267).

Además, aportes como el de Rutter (2005, p.14), son sin duda significativos, él considera que 
"en la medida en que las conductas son influenciadas genéticamente (y casi todas las conductas son influenciadas genéticamente en alguna extensión dado que el trabajo de la mente está basado en el funcionamiento del cerebro, y los genes influyen en el desarrollo y la estructura del cerebro así como hacen con otros órganos del cuerpo), la existencia de las correlaciones gene-ambiente significan que los factores genéticos van a jugar algún papel (en una forma de contribución, más que de determinación) en la afectación de las diferencias individuales en la vivencia de diferentes experiencias de vida".

Como otro ejemplo del cambio conceptual que se experimenta, Bell, Romano y Flynn (2015) dentro de su perspectiva sistémica y dinámica de la resiliencia destacan la existencia de distintos niveles que se influyen mutuamente de acuerdo con los grados de proximidad al individuo: elementos que ubican en un microsistema, otros en un exosistema y un nivel más externo denominado macrosistema; destacan que, en conjunto, sus interacciones inciden en la expresión de la resiliencia.

Ya en la actualidad, investigaciones como la de Liu, Reed y Girard (2017), examinan la naturaleza dinámica del proceso de resiliencia, planteando que incluye la interacción entre los individuos y sus contextos socioecológicos y proponiendo un modelo multisistémico que considera variables intraindividuales, interpersonales y socioecológicas. Estos autores enfatizan en la resiliencia como un proceso interactivo, dinámico y multidimensional por naturaleza.

Desde una perspectiva similar, McLaughlin y Lambert (2017) presentan un modelo biopsicosocial de la resiliencia, en el que contemplan tanto los mecanismos individuales de procesamiento de la información de los retos ambientales, como las redes de apoyo que pueden funcionar a manera de activadores de los procesos neurobiológicos que se activan ante una situación de adversidad.

Algunos de los aspectos más importantes de esta perspectiva de la resiliencia como proceso dinámico son: el énfasis en la multiplicidad de elementos que intervienen y las interrelaciones e interdependencia, siempre cambiantes, que se dan entre los individuos y los sistemas sociales, así como el reconocimiento de que la resiliencia no es la ausencia de vulnerabilidad, ni tampoco una característica inherente a la personalidad (Lavoie, Pereira y Talwar, 2016; Waller, 2001); y el destacar que dichas relaciones deben ser tomadas en cuenta, dado que la resiliencia es un proceso constructivo que se elabora en el transcurso de la existencia (Poilpot, 2004; Van Kessel, 2013).

$\mathrm{Al}$ abordar la resiliencia como proceso dinámico, se destaca además el hecho de que la interacción entre los sistemas, individuales y colectivos no es pasiva ni meramente receptora, sino activa, dinámica y de mutua afectación (Kim-Cohen, Moffitt, Caspi y Taylor, 2004; Liu, Reed y Girard, 2017; Reghezza-Zitt, Lhomme y Provitolo, 2015) y se extiende la consideración de la resiliencia a toda y cualquier momento en la historia de vida, no solo a la niñez o adolescencia, que predominantemente ha sido el foco de atención e investigación (Rodríguez-Fernández et ál. 2016; Roisman, 2005; van Kessel, 2013).

Desde la perspectiva dinámica, se considera la presencia de multidimensionalidad (es decir, que puede mostrarse mejor adaptación en unas áreas o situaciones que en otras), de una alta variabilidad en diferentes ámbitos para una misma persona y de variaciones en las características de interacción con el medio, a través del tiempo (Lavoie, Pereira y Talwar, 2016; Lawford y Eiser, 2001; Luthar, Cicchetti y Becker, 2000; Waller, 2001), así como la reafirmación de que la resiliencia "resulta de un proceso dinámico, evolutivo... fruto de la interacción entre el propio individuo y su entorno, entre las huellas de sus vivencias anteriores y el contexto del momento en materia política, económica, social o humana" (Manciaux, Vanistendael, Lecomte y Cyrulnik, 2003, p.23).

De esta manera, el conocimiento apunta a que lo que ha sido denominado resiliencia en el ser humano, tiene sus raíces en un proceso coevolutivo, como coevolutivo y dinámico es el desarrollo humano, y que dada la multiplicidad de factores que intervienen, no es posible establecer juicios predictores ni predestinadores de historias de vida en cuanto a las características adaptativas y el curso que tendrá una biografía. Como demandan Manciaux, Lecomte, Vanistendael y Schweizer (2003, p.305), esta perspectiva de la resiliencia lleva a "dejar la idea de la fatalidad... de un determinismo que juzgue que 'todo está decidido'... tras un suceso dado". Implica, además, como comenta Puerta (2002), eliminar cualquier vinculación con la teoría 
de la tabula rasa, considerar el potencial de todo individuo y dejar a un lado la creencia de que existen seres totalmente desprotegidos y vulnerables ante la fuerza de un evento que en sí mismo puede afectar el desarrollo de esa persona.

Una vez reconocido el dinamismo de la coevolución entre la persona y su medio, los nuevos planteamientos en la investigación sobre resiliencia, se dirigen hacia el entendimiento de cómo funcionan y se desarrollan los sistemas adaptativos en el ser humano, y cómo estos responden a las variaciones en el ambiente, es decir, de qué manera el individuo, como un complejo sistema viviente, se relaciona a lo largo del tiempo con el entorno en el cual está inmerso.

El solo hecho de que poco a poco se reconozca que el desarrollo humano no se da en el vacío, sino que es producto de continuas transacciones entre individuos y sus ambientes físicos, culturales y sociales en los cuales crece, es ya un avance en la comprensión de que este, "es un continuo proceso de adaptación y acomodación entre individuos y sus entornos" (Waller, 2001, p.290). Sin embargo, la disociación con la neurociencia y la pedagogía aún continúa reinando en la investigación en resiliencia, y son pocas las referencias que en esta última disciplina, contemplan siquiera que el cerebro humano existe y que es modificado por las relaciones que el individuo establece con su medio.

Aun así, se aprecian intentos por empezar a achicar la brecha, y la llamada de atención que hacía Kaplan (1999, citado por Mayhew y Mayhew, 2003, p.267) acerca de que los científicos deberían "centrarse en la elaboración de modelos teóricos que integren los factores individuales, ambientales y situacionales, que se influyen e interaccionan, e influyen también de varios modos en diversas variables, en distintas etapas del desarrollo observado en medios sociales diversos", parece comenzar a tomarse en cuenta, por lo menos en la ampliación del campo de visión hacia la multiplicidad de elementos que potencialmente pueden incidir en una escritura biográfica.

\section{Algunas observaciones con respecto aL panorama teórico-Conceptual y EMPÍRICO DE LA RESILIENCIA}

Luego de realizar un repaso acerca del confuso panorama conceptual de la resiliencia, de sus orígenes y sus abordajes en las ciencias sociales, se considera importante señalar algunos aspectos:

Siendo un concepto utilizado inicialmente en áreas de la metalurgia y la osteología, su aplicación a las ciencias sociales constituye, como describe Roisman (2005), un constructo científico fraguado de trampas y de promesas potenciales. Lo escrito sobre el tema, hasta el momento responde en su mayoría ni más ni menos que a un constructo social básicamente occidental, que interpreta lo que el sistema nervioso humano ha venido haciendo evolutivamente: desarrollar, adquirir y fortalecer habilidades para enfrentar condiciones adversas, analizarlas consciente o inconscientemente, y responder al entorno circundante.

Desde los elementos genéticos más básicos, como es la amplitud única de la oposición del pulgar, que permite la prensión de pinza y la ejecución de innumerables acciones, hasta la elaboración del vestido y la construcción de viviendas en procura de adaptarse y sobrevivir ante situaciones ambientales muy diversas, el desarrollo del ser humano ha venido escribiéndose biológicamente con una predisposición impresionante para enfrentarse a su entorno, vivir experiencias retadoras, aprender y, de ser posible, coevolucionar con su ecología circundante.

Desde este punto de vista, el cual es el que se propone en el presente trabajo, lo que es posible señalar como indispensable para la resiliencia como proceso humano básico de la evolución, es la existencia del encéfalo y la oportunidad de acceder a vivencias diversas que generen los retos y oportunidades necesarios, para que dicho sustrato tenga la posibilidad de construir, deconstruir y reconstruir las características interactivas con su entorno procurando la adaptación y la evolución.

La resiliencia como proceso dinámico identificado desde la perspectiva de los científicos sociales, requiere de la integración del conocimiento neurocientífico para detener la caída en una especie de espiral 
interminable en la que la disociación entre las ciencias biológicas y las ciencias sociales, es patente en cuanto a la ambigüedad y discordancia conceptual se refiere.

Aun cuando se haga referencia al desarrollo humano y a los factores que pueden incidir de formas distintas en la escritura de una historia de vida, la ausencia de la concepción de ser humano, como ser neurobiológico en evolución con un sistema nervioso complejo y plástico, socava cualquier intento de aproximarse a la comprensión de la resiliencia y repercute en que el entendimiento de la influencia de las experiencias de vida en el devenir biográfico, continúe teniendo lagunas importantes.

Sin una base neurocientífica como sustento, la gran mayoría de observaciones en el tema de la resiliencia no serán más que elucubraciones, es decir, divagaciones complicadas y con apariencia de profundidad, pero ausentes de un fundamento teórico que dé cuenta de cómo el ser humano coevoluciona con el entorno, de cómo se da el desarrollo epigénico de un sustrato neurobiológico básico, de cómo cada individuo percibe los sistemas que le rodean, forma memorias, aprende y desarrolla también funciones cognitivas y neuropsicológicas, procesos todos, mediante los cuales siente al mundo, le enfrenta y puede hacerse sentir en él.

Y tal vez el momento de encontrar una respuesta satisfactoria aun esté lejos, lo que sí, es que actualmente se maneja gran cantidad de información acerca del potencial de afectación que tiene el entorno, al entrar en coevolución con un organismo en desarrollo, y parece plausible conciliar el conocimiento actual en neurodesarrollo, con el aporte que han brindado las ciencias sociales, y acercarse a la comprensión de cómo la coevolución entre un ser humano con un sustrato neurobiológico particular y las experiencias de vida a las que accede en un entorno que responde hacia él también de manera singular, van delineando los estados mentales, de consciencia y conducta que el medio social interpreta como resilientes o no resilientes.

Sin duda el concepto de resiliencia continúa en construcción, como en proceso está también el conocimiento acerca del desarrollo humano; sin embargo no es productivo ni funcional desligar ambas ramas, ni es válido tampoco, intentar interpretar expresiones de la mente, de la consciencia y de la conducta, sin partir del hecho de que estas son generadas por un sistema nervioso que está inmerso en un ambiente particular.

Todos los niños nacen cableados para sentir, dispuestos para aprender y virtualmente cada aspecto del desarrollo temprano, desde la circuitería cerebral, a la emergencia de la capacidad para resolver problemas y experimentar empatía, es afectado por la influencia dinámica y constante de las experiencias de vida y por la naturaleza y calidad de relaciones que se establecen con ese ser humano. No hay duda que al respecto, el extendido debate de naturaleza versus crianza es científicamente obsoleto.

\section{Propuesta de enfoque de resiliencia, una perspectiva neurobiológica y COEVOLUTIVA}

Si se parte de la consideración de las bases neurobiológicas del proceso de desarrollo humano y de la existencia de la evolución, de la plasticidad y de la epigénesis como rasgos fundamentales del papel, tintas y plumas con que se escriben las historias de vida, es posible empezar a ver la resiliencia no solo como un constructo social, sino pasar a analizarla como parte de un proceso neurobiológico y coevolutivo que permite la diversidad, la supervivencia de la especie y la exploración y comprensión de la afectación mutua entre organismo y entorno.

Si se parte de la consideración de las bases neurobiológicas del proceso de desarrollo humano y de la existencia de la evolución, de la plasticidad y de la epigénesis como rasgos fundamentales del papel, tintas y plumas con que se escriben las historias de vida, es posible empezar a ver la resiliencia no solo como un constructo social, sino pasar a analizarla como parte de un proceso neurobiológico y coevolutivo que permite la diversidad, la supervivencia de la especie y la exploración y comprensión de la afectación mutua entre organismo y entorno.

De esta manera, se propone abordar la resiliencia como: 
Un proceso dinámico, de aprendizaje no solo del individuo sino también del entorno con el cual se encuentra en estrecha coevolución, en donde el acceso al nutrimento que conlleva la interacción social como elemento de mutua afectación, junto a los retos generados en dicha coevolución, le proporciona al individuo en, y con su ambiente, la posibilidad de enfrentar situaciones de adversidad, aprender de ellas y utilizar dicha experiencia para desarrollar estrategias de enfrentamiento ante situaciones posteriores.

Todos los niños nacen cableados para sentir, dispuestos para aprender y virtualmente cada aspecto del desarrollo temprano, desde la circuitería cerebral, a la emergencia de la capacidad para resolver problemas y experimentar empatía, es afectado por la influencia dinámica y constante de las experiencias de vida y por la naturaleza y calidad de relaciones que se establecen con ese ser humano. No hay duda que al respecto, el extendido debate de naturaleza versus crianza es científicamente obsoleto.

Desde la perspectiva que se propone, la resiliencia, como parte del proceso de neurodesarrollo, es un fenómeno epigénico evolutivo, que requiere de la coevolución entre el sustrato neural y el entorno, hecho indispensable para el desarrollo humano y para el aprendizaje.

Dado que el sistema educativo se constituye en un actor indiscutible de las escrituras biográficas especialmente en los períodos de la infancia y la adolescencia, su papel como promotor o limitante de la resiliencia adquiere una especial relevancia.

La resiliencia, como menciona Lecomte (2003, p.202) "no significa volver a empezar de cero, sino aprender de la experiencia y sacar de ella lecciones de vida". La orientación que puede ofrecerse en los centros educativos, es un elemento de incalculable valía para este aprendizaje individual y colectivo.

Precisamente, el aprendizaje es destacado por autores como Puerta (2002) y Vaillant (2004), quienes consideran que un aspecto importante ante la vivencia de situaciones adversas, es la posibilidad de adjudicar sentido a estas, organizar con ellas un relato coherente, identificar el espacio para proyectar las propias capacidades y construir. En este sentido, el entorno escolar, con los mediadores que en él se encuentran, adquiere también un papel preponderante en la posibilidad de que el estudiantado aprenda de las situaciones retadoras y utilice dicha experiencia para desarrollar estrategias de afrontamiento efectivas.

La plasticidad, característica intrínseca del sistema nervioso, le permite al organismo modificarse a través de las experiencias de vida; dicha cualidad constituye quizás uno de los argumentos principales no solo para demostrar que todo individuo tiene el potencial de aprender y por lo tanto de aprovecharse y nutrirse de la interacción con su medio, sino también, para concienciar acerca de que ese "entrenamiento diario" con la cotidianidad tanto dentro como fuera de la escuela, puede promover o limitar procesos fundamentales que van desde la gliogénesis y neurogénesis, a la mielinización, la excitabilidad de las membranas sinápticas, el número y funcionamiento de los canales iónicos, las características y cantidades de neurotransmisores disponibles, el establecimiento de sinapsis, los cambios axonales y dendríticos, el grado de contacto entre la glia y la sinapsis, la formación de circuitos y la apoptosis.

En la actualidad, la evidencia en neurodesarrollo, neurociencias y neuropsicología soporta el hecho de que la respuesta del medio ante un ser humano que exprese un fenotipo bioestructural conductual y social particular, dependiendo de los constructos sociales que lo dirijan, puede promover o limitar el desarrollo y la expresión dinámica de ese proceso de aprendizaje base para la resiliencia, incidiendo en las características de las respuestas adaptativas que se generen. Este hecho constituye una base fundamental que agrega un soporte neurocientífico para la toma de decisiones que promueven la inclusión en el sistema educativo.

Es así, que dentro de un enfoque coevolutivo y epigénico, el tomar conciencia de la resiliencia como proceso de aprendizaje en donde interacción social como elemento de mutua afectación, junto a los retos generados en dicha coevolución, le proporciona al individuo en y con su ambiente la posibilidad de aprender y utilizar dicha experiencia para enfrentarse a situaciones posteriores, implica no limitar dicha responsabilidad al individuo, sino reconocer que este forma parte de una colectividad cuya resiliencia es fundamental para el desarrollo de todos sus miembros. 


\section{REFERENCIAS}

Agaibi, C. \& Wilson, J. (2005). Trauma, PTSD, and Resilience, A Review of the Literature. Trauma, Violence, \& Abuse. 6 (3), 195-216.

Bell, T., Romano, E. y Flynn, R. (2015). Profiles and predictors of behavioral resilience among children in child welfare. Child Abuse \& Neglect. 48, 92-103

Benard, B. (1996). El fomento de la resiliencia en los niños. ERIC Digest. EDO-PS-96-1, 1-3.

Block, J. \& Block, J. (1980). The role of ego-control and ego-resiliency in the organization of behavior. En W. Andrew Collins (Ed.). Development of cognition, affect, and social relations. The Minnesota Symposia on Child Psychology (pp. 39 - 101). Hillsdale, NJ: Erlbaum.

Block, J. \& Kremen, A. (1996). IQ and Ego-Resiliency: Conceptual and Empirical Connections and Separateness. Journal of Personality and Social Psychology. 70 (2), 349-361.

Born, M. y Boët, S. (2004). La resiliencia ilegal. En Cyrulnik, B., Tomkiewicz, S., Guénard, T., Vanistendael, S., Manciaux, M. y otros. El realismo de la esperanza, Testimonios de experiencias profesionales en torno a la resiliencia (pp.171-184). Barcelona: Editorial Gedisa.

Bosworth, K. \& Earthman, E. (2002). From theory to practice: school leaders perspectives on resiliency. Journal of Clinical Psychology. 58 (3), 299-306.

Bouvier, P. (2003). Temperamento, riesgo y resiliencia en el niño. En Manciaux, M. (comp.). La resiliencia: resistir y rehacerse. (pp.63-74). Barcelona: Editorial Gedisa, S.A.

Carazo, V. (2013). Neurodesarrollo, Diversidad y Segregación, un acercamiento teórico a sus bases neuropsicológicas. Tesis Doctoral. Universidad de Salamanca, España.

Carazo, V. y López, L. (2009). "Aprendizaje, coevolución neuroambiental”. Coordinación Educativa y Cultural Centroamericana (CECC). San José, Costa Rica.

Decreto Ejecutivo 40955. Diario Oficial La Gaceta. San José, Costa Rica 19 de marzo del 2018. Recuperado de: http://196.40.56.11/scij/Busqueda/Normativa/Normas/nrm_norma.aspx? param $1=$ NRM\&nValor $1=1 \&$ nValor2=86181\&nValor3=111664\&strTipM=FN

Dubowitz, H., Thompson, R., Proctor, L., Metzger, R., Black, M., English, D., Poole, G. \& Magder, L. (2016). Adversity, Maltreatment, and Resilience in Young Children. Academic Pediatrics.16:233-239

Eisenberg, N., Spinrad, T., Fabes, R., Reiser, M., Cumberland, A., Shepard, S., Valiente, C., Losoya, S., Guthrie, I. \& Thompson, M. (2004). The Relations of Effortful Control and Impulsivity to Children's Resiliency and Adjustment. Child Development. 75 (1), 25 - 46.

Engle, P., Castle, S. \& Menon, P. (1996). Child development: vulnerability and resilience. Soc. Sci. Med. 43, (5), 621-635.

Fergus, S. \& Zimmerman, M. (2005). ADOLESCENT RESILIENCE: A Framework for Understanding Healthy Development in the Face of Risk. Annual review of public health. 26, 399-419.

Finley, M. (1994). Cultivating resilience: an overview for rural educators and parents. ERIC Digest. EDO-RC-94-5, $1-4$.

Gottesman, I. \& Hanson, D. (2005). Human Development: Biological and Genetic Processes. Annual Reviews of Psychology. 56, 263-286.

Hunter, A. \& Chandler, G. (1999). Adolescent Resilience. Journal of Nursing Scholarship. 31 (3), 243-247.

Jablonka, E. y Lamb, M. (2002). The changing concept of epigenetics. Annals of the New York Academy of Sciences. 981, 82-96.

Jacelon, C. (1997). The trait and process of resilience. Journal of Advanced Nursing. 25, 123-129.

Kelley, T. (2005). Natural Resilience and Innate Mental Health. American Psychologist. 60 (3), 265.

Kim-Cohen, J., Moffitt, T., Caspi, A. y Taylor, A. (2004). Genetic and environmental processes in young children's resilience and vulnerability to socio-economic deprivation. Child Development. 75 (3), 651-668. 
Lavoie, J., Pereira, L. y Talwar, V. (2016). Children's Physical Resilience Outcomes: Meta-Analysis of Vulnerability and Protective Factors. Journal of Pediatric Nursing. 31, 701-711.

Lawford, J. y Eiser, C. (2001). Exploring links between the concepts of Quality of Life and Resilience. Pediatric Rehabilitation. 4 (4), 209-216.

Lecomte, J. (2003). El buen uso de la memoria y del olvido. En Manciaux, M. (comp.). La resiliencia: resistir y rehacerse. (pp.202-213). Barcelona: Editorial Gedisa, S.A.

Liu, J., Reed, M.y Girard, T. (2017) Advancing resilience: An integrative, multi-system model of resilience. Personality and Individual Differences. 111, 111-118.

López, L. (2005, Setiembre). Resiliencia humana: del constructo social a sus bases neurobiológicas. Trabajo presentado en el XV Simposio Costarricense de Psicobiología y III Simposio de Psicología Cognitivo Conductual, de la Universidad de Costa Rica. San José, Costa Rica.

Luthar, S., Cicchetti, D. y Becker, B. (2000). The construct of resilience: a critical evaluation and guidelines for future work. Child Development. 71 (3): 543-562.

Manciaux, M. (2004). Conclusiones y perspectivas. En Cyrulnik, B., Tomkiewicz, S., Guénard, T., Vanistendael, S., Manciaux, M. y otros. El realismo de la esperanza, Testimonios de experiencias profesionales en torno a la resiliencia (pp.225-233). Barcelona: Editorial Gedisa, S.A.

Manciaux, M., Vanistendael, S., Lecomte, J. y Cyrulnik, B. (2003). La resiliencia: estado de la cuestión. En Manciaux, M. (comp.). La resiliencia: resistir y rehacerse. (pp.17-27). Barcelona: Editorial Gedisa, S.A.

Mandleco, B. y Peery, C. (2000). An organizational framework for conceptualising resilience in children. Journal of Child and Adolescent Psychiatric Nursing. 13 (3), 99-111.

Masten, A. (2001). Ordinary magic, resilience processes in development. American Psychologist. 56 (3), 227-238.

Mayhew, K. y Mayhew, M. (2003). La resiliencia en Estados Unidos: desarrollos recientes y aplicaciones prácticas. En Manciaux, M. (comp.). La resiliencia: resistir y rehacerse. (pp.265-281). Barcelona: Editorial Gedisa.

McLaughlin, K. y Lambert, H. (2017). Child trauma exposure and psychopathology: mechanisms of risk and resilience. Current Opinion in Psychology. 14, 29-34

Olsson, C., Bond, L., Burns, J.,Vella-Brodrick, D. y Sawyer, S. (2003). Adolescent resilience: a concept analysis. Journal of Adolescence. 26, 1-11.

Pedrot, P. (2004). El reconocimiento social y jurídico de los traumas. En Cyrulnik, B.,Tomkiewicz, S., Guénard, T., Vanistendael, S., Manciaux, M. y otros. El realismo de la esperanza, Testimonios de experiencias profesionales en torno a la resiliencia (pp.237-240). Barcelona: Editorial Gedisa.

Poilpot, M. (2004). La resiliencia: el realismo de la esperanza. En Cyrulnik, B., Tomkiewicz, S., Guénard, T., Vanistendael, S., Manciaux, M. y otros. El realismo de la esperanza, Testimonios de experiencias profesionales en torno a la resiliencia (pp.11-14). Barcelona: Editorial Gedisa.

Proag, V. (2014). The concept of vulnerability and resilience. Procedia Economics and Finance. 18, 369 - 376.

Puerta, M. (2002). Resiliencia. La estimulación del niño para enfrentar desafíos. Argentina: Grupo Editorial Lumen.

Rak. C. (2002). Heroes in the Nursery: Three case studies in resilience. Journal of Clinical Psychology. 58 (3), 247-260.

Reghezza-Zitt, M, Lhomme, S. \& Provitolo, D. (2015). Defining Resilience: When the Concept Resists. (pp.1-27) En: Resilience Imperative Uncertainty, Risks and Disasters. ISTE Press - Elsevier.

Robinson, J. (2000). Are there implications for prevention research from studies of resilience? Child Development. $71(3), 570-572$.

Rodríguez-Fernández, A., Ramos-Díaz, E., Ros, I., Fernández-Zabala, A. y Revuelta. L. (2016). Bienestar subjetivo en la adolescencia: el papel de la resiliencia, el autoconcepto y el apoyo social percibido. Suma Psicológica 23, 60-69.

Roisman, G. (2005). Conceptual Clarifications in the Study of Resilience. American Psychologist. 60 (3), 264-265.

Rutter, M. (2005). Environmentally mediated risks for psychopathology: research strategies and findings. Journal of the American Academy of Child and Adolescent Psychiatry. 44 (1), 3-18.

Sanes, D., Reh, T. \& Harris, W. (2000). Development of the Nervous System. United States of América: Academic Press. 
Stein, H., Fonagy, P., Ferguson, K. \& Wisman, M. (2000). Lives through time: an ideographic approach to the study of resilience. Bulletin of the Menninger Clinic. 64 (2), 281-305.

Theis, A. (2003). La resiliencia en la literatura científica. En M. Manciaux, (Comp.). La resiliencia: resistir y rehacerse. (pp.45-59). Barcelona: Editorial Gedisa.

Titran, M. (2004). ¿Son resilientes nuestras instituciones? B. Cyrulnik, S. Tomkiewicz, T. Guénard, S. Vanistendael, M. Manciaux y otros. El realismo de la esperanza, Testimonios de experiencias profesionales en torno a la resiliencia (pp.241-243). Barcelona: Editorial Gedisa, S.A.

Tomkiewicz, S. (2004). El surgimiento del concepto. En B. Cyrulnik, S. Tomkiewicz, T. Guénard, S. Vanistendael, M. Manciaux, y otros. El realismo de la esperanza, Testimonios de experiencias profesionales en torno a la resiliencia (pp.33-50). Barcelona: Editorial Gedisa, S.A.

Turgade, M. y Fredrickson, B. (2004). Resilient Individuals Use Positive Emotions to Bounce Back From Negative Emotional Experiences. Journal of Personality and Social Psychology. 86 (2):320-333.

Vaillant, M. (2004). Reciclaje de la violencia y capacidad de resiliencia: la hipótesis transicional en la reparación. En B. Cyrulnik, S. Tomkiewicz, T. Guénard, S. Vanistendael, M. Manciaux, y otros (Aut.). El realismo de la esperanza, Testimonios de experiencias profesionales en torno a la resiliencia (pp.185-196). Barcelona: Editorial Gedisa.

Van Kessel, G. (2013). The ability of older people to overcome adversity: A review of the resilience concept. Geriatric Nursing. 34, 122-127

Walker, F., Pfingst, K., Carnevali, L., Sgoifo, A., Nalivaik, E. (2017). In the search for integrative biomarker of resilience to psychological stress. Neuroscience and Biobehavioral Reviews 74, 310-320.

Waller, M. (2001). Resilience in Ecosystemic Context: Evolution of the Concept. American Journal of Orthopsychiatry. 71(3), 290-297.

Werner, E. (2004). Journeys from childhood to midlife: risk, resilience, and recovery. Pediatrics. 114 (2), 492.

Werner, E., Bierman, J., French, F., Simonian, K., Connor, A., Smith, R. \& Campbell, M. (1968). Reproductive and environmental casualties: a report on the 10-year follow-up of the children of the Kauai Pregnancy Study. Pediatrics. 42 (1), 112-127.

Werner, E., Honzik, M. \& Smith, R. (1968). Prediction of intelligence and achievement at ten years from twenty months pediatric and psychologic examinations. Child Development. 39, 1063-1075.

\section{BY-NC-ND}

